\title{
Study of cosmic ray propagation using GALPROP with observation data
}

\author{
Hongyi $\mathbf{W} \mathbf{u}^{\mathbf{1}}$ \\ Univ. of Maryland College Park \\ Inst. for Phys. Sci. and Tech., University of Maryland, College Park, MD 20742, USA \\ E-mail: hwuleterpmail.umd.edu
}

\section{Eun-Suk Seo}

Univ. of Maryland College Park

Inst. for Phys. Sci. and Tech., University of Maryland, College Park, MD 20742, USA

E-mail: seo@umd. edu <seo@umd. edu>

\section{Vladimir Ptuskin}

IZMIRAN, Moscow

IZMIRAN, Troitsk, Moscow 108840, Russia

E-mail: vptuskinehotmail.com

The high-accuracy measurements of protons and nuclei energy spectra in the cosmic ray experiment AMS confirmed the earlier experimental results of ATIC-2, CREAM and PAMELA measurements on the presence of spectral hardening at magnetic rigidity about $200 \mathrm{GV}$. The AMS data on the secondary nuclei $\mathrm{Li}, \mathrm{Be}$ and $\mathrm{B}$ indicated that the hardening could be due to the transition to weaker rigidity dependence of cosmic ray confinement time in the Galaxy above 200 GV. However, the possible hardening of the source spectrum is also not excluded. To study the origin of spectrum hardening, we use the numerical code GALPROP to compute the propagation of cosmic rays under different assumptions on the rigidity dependence of cosmic ray source and propagation parameters in a few Galactic models.

36th International Cosmic Ray Conference -ICRC2019-

July 24th - August 1st, 2019

Madison, WI, U.S.A.

\section{${ }^{1}$ Presenter, undergraduate student}




\section{Introduction}

Recent high-accuracy measurements of cosmic ray energy spectrum have revealed spectral deviation from the plane power law at magnetic rigidities $200-300 \mathrm{GV}$. The presence of spectral hardening was found in calorimeter experiments ATIC-2 ${ }^{1}$ and CREAM $^{2}$ on baloons, and with higher precision in magnetic spectrometer experiments PAMELA ${ }^{3}$ and $\mathrm{AMS}^{4}$. The change of the power law exponent by $\delta_{\gamma} \approx 0.1$ is established now for protons, primary and secondary nuclei. The origin of this phenomenon is uncertain and may reflect the corresponding breaks of the source spectrum or/and diffusion coefficient, or the effect of nearby source, see Serpico ${ }^{5}$ for discussion. In the present work we analyze CREAM and AMS data on cosmic ray protons; $\mathrm{He}, \mathrm{Li}, \mathrm{Be}, \mathrm{B}, \mathrm{C}$, O nuclei; electrons and antiprotons in two diffusion models with breaks in the source spectrum and diffusion coefficient. It is found that adding a break in the diffusion coefficient, which is changing the diffusion coefficient index from 0.494 to 0.30 at a rigidity of $200 \mathrm{GV}$, is a preferred method, while adding a break in the source spectra can not adequately explain all observations.

\section{Model}

The GALPROP ${ }^{6}$ is a numerical code for calculating the propagation of relativistic charged particles. The contents include diffusive reacceleration and nuclear spallation, secondary particle production, radioactive decay, electron interactions and so on. ${ }^{7}$ The equation shown in Figure 1 is the main equation GALPROP solves numerically:

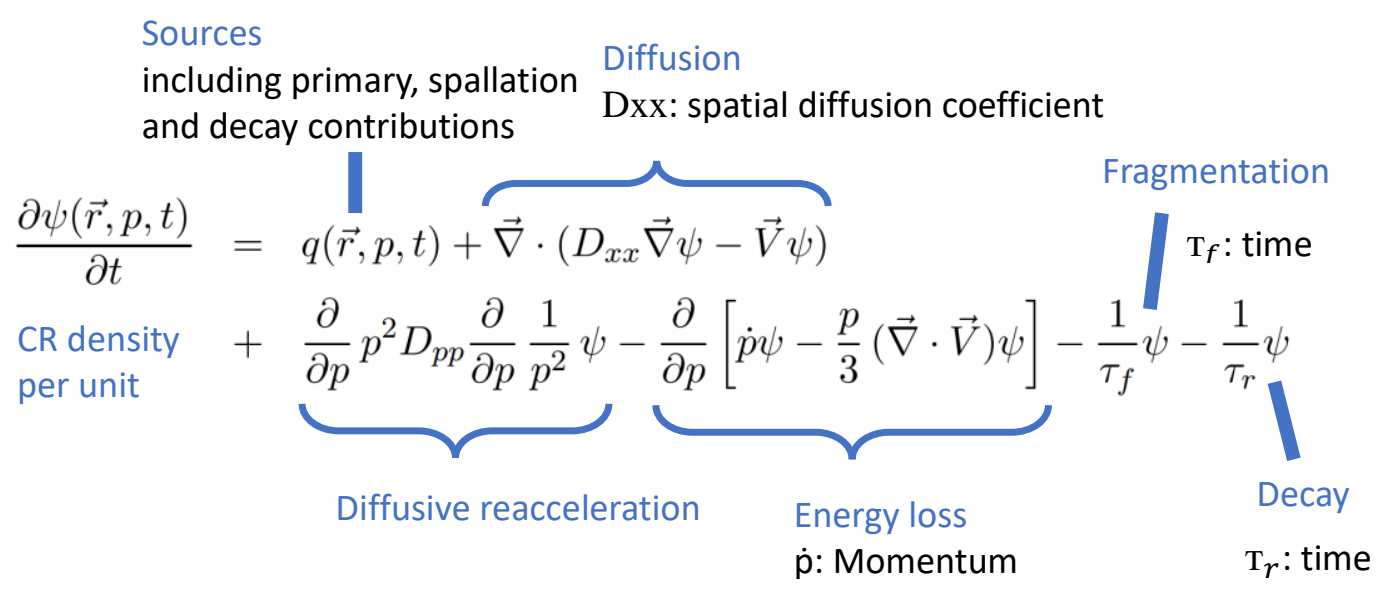

Figure. 1 Equation for the propagation of cosmic ray used in GALPROP ${ }^{l}$

The diffusion coefficient relates to rigidity in the form: $D \propto \beta D_{0 x x} R^{-D_{g}}$, where $\mathrm{D}$ is the diffusion coefficient, $D_{g}$ is the diffusion coefficient index. The computation provides one rigidity break where the diffusion coefficient indices can be different above and below it, and the diffusion coefficient will be normalized at the rigidity break. The source spectra also pose an exponential relation to rigidity in the form: $\Phi \propto R^{-\gamma}$, where $\gamma$ is the nucleus injection index. Similar to how GALPROP handles diffusion coefficient, we can have a number of rigidity breaks in the computation with different nucleus injection indices above and below them. We consider diffusion models with static cosmic ray halo of the size $\mathrm{H}=4 \mathrm{kpc}$ and set $\mathrm{V}=0$. 
In this study, we use GALPROP to study the propagation of cosmic rays based on an approach to fit $\mathrm{B} / \mathrm{C}$ ratio and specific element spectra from current data with computational curves produced by GALPROP.

\subsection{Default Run}

We obtained default parameters from the GALPROP WebRun (version 54), which can be found at https://galprop.stanford.edu/webrun.php. The version of GALPROP we use is v56, thus we are changing the default parameter set according to a newer paper corresponding to our version. These basic parameters are adjusted the parameters according to a paper by Porter et al., ${ }^{7}$ which uses GALPROP v56 for a complete run before the version is released. The parameters are listed in Table 1 . Set 0 is the parameter set we are using as the default setting, where there are no second breaks in source spectra, and no breaks in diffusion coefficient. These parameters overwrite those in the default parameter set from GALPROP v54 WebRun to fit the new version. [a] The diffusion coefficient is normalized to $\mathrm{D}_{0, \mathrm{xx}}$ at $4 \mathrm{GV}$. [b] The nucleus injection indices of $\mathrm{He}$ are produced by subtracting $\Delta_{\mathrm{He}}$ from each of the proton injection indices $\gamma_{0, \mathrm{H}}, \gamma_{1, \mathrm{H}}$, and $\gamma_{2, \mathrm{H}}$. [c] The proton flux and electron flux are normalized at the solar location at a kinetic energy of $100 \mathrm{GeV}$ per nucleon.[d] The injection spectra for the isotopes listed below are adjusted as a ratio of the proton injection spectrum at $100 \mathrm{GeV}$. All other unmentioned spectra are the same as the WebRun. [e] The solar modulation of AMS is used in this run to fit data from AMS and CREAM.

Table 1. Set 0 parameters with no extra breaks in diffusion coefficient and source spectra

\begin{tabular}{cc|cc}
\hline Parameter & Set 0 & Parameter & Set 0 \\
\hline $\mathrm{D}_{0, \mathrm{xx}}\left[10^{28} \mathrm{~cm}^{2} \mathrm{~s}^{-1}\right]^{\mathrm{a}}$ & 4.37 & $\gamma_{1, \mathrm{e}}$ & 2.765 \\
$\delta$ & 0.494 & $\mathrm{R}_{1, \mathrm{e}}[\mathrm{GV}]$ & 5.95 \\
$\mathrm{v}_{\mathrm{A}}\left[\mathrm{km} \mathrm{s}^{-1}\right]$ & 7.64 & $\mathrm{~J}_{\mathrm{p}}\left[10^{-9} \mathrm{~cm}^{-2} \mathrm{~s}^{-1} \mathrm{sr}^{-1} \mathrm{MeV}^{-1}\right]^{\mathrm{c}}$ & 4.598 \\
$\gamma_{0}$ & 1.47 & $\mathrm{~J}_{\mathrm{e}}\left[10^{-11} \mathrm{~cm}^{-2} \mathrm{~s}^{-1} \mathrm{sr}^{-1} \mathrm{MeV}^{-1}\right]^{\mathrm{c}}$ & 1.221 \\
$\gamma_{1}$ & 2.366 & $\mathrm{q}_{0}{ }^{4}{ }_{\mathrm{He}} / \mathrm{q}_{0, \mathrm{p}} \times 10^{-6 \mathrm{~d}}$ & 93892 \\
$\mathrm{R}_{1}[\mathrm{GV}]$ & 3.64 & $\mathrm{q}_{0}{ }^{12}{ }^{12} \mathrm{~d} / \mathrm{q}_{0, \mathrm{p}} \times 10^{-6 \mathrm{~d}}$ & 2882 \\
$\gamma_{0, \mathrm{H}}$ & 1.74 & $\mathrm{q}_{0}{ }^{12}{ }^{\mathrm{o}} / \mathrm{q}_{0, \mathrm{p}} \times 10^{-6 \mathrm{~d}}$ & 3780 \\
$\gamma_{1, \mathrm{H}}$ & 2.350 & $\mathrm{q}_{0}{ }^{20}{ }_{\mathrm{Ne}} / \mathrm{q}_{0, \mathrm{p}} \times 10^{-6 \mathrm{~d}}$ & 356 \\
$\mathrm{R}_{1, \mathrm{H}}[\mathrm{GV}]$ & 5.78 & $\mathrm{q}_{0,}{ }^{24}{ }_{\mathrm{Mg}} / \mathrm{q}_{0, \mathrm{p}} \times 10^{-6 \mathrm{~d}}$ & 644 \\
$\Delta_{\mathrm{He}}{ }^{\mathrm{b}}$ & 0.026 & $\mathrm{q}_{0}{ }^{28}{ }_{\mathrm{Si}} / \mathrm{q}_{0, \mathrm{p}} \times 10^{-6 \mathrm{~d}}$ & 742 \\
$\gamma_{0, \mathrm{e}}$ & 1.63 & $\Phi_{\mathrm{AMS}}[\mathrm{MV}]$ & 610 \\
\hline
\end{tabular}

According to the AMS data, the spectra of antiproton, Lithium, Beryllium, Boron, Carbon, and Oxygen all show a significant hardening at high energies, starting at $\sim 200 \mathrm{GV}$ in rigidity. This discrepancy doesn't agree with the exponential model of diffusion coefficient we use in the default model. Therefore, in addition to the default run (set 0 ), we apply two other parameter sets to try to fit the hardenings while keeping the other parts of the spectra within acceptable range.

\subsection{Methods and Verification}

To fit the data, we try to approach through two methods: 
1. Diffusion coefficient. Adding a break where we change the diffusion coefficient $D$ to adjust the curves of element spectra. This also allows us to adjust the $\mathrm{B} / \mathrm{C}$ ratio closer to the data curve.

2. Source spectra. Changing the source spectra at high energy can also adjust the curves of element spectra. We will adjust the source spectra of specific elements such as proton, Helium, and electron as required instead of affecting all particles all at once.

These two methods are applied to the model with the following two sets of parameter change:

Set 1: This computation adds a rigidity break at $200 \mathrm{GV}$ and has a change of diffusion coefficient from 0.494 to 0.30 after the break as summarized in Table 2. A break is added to the diffusion coefficient at a rigidity of $\mathrm{D}_{\text {rigid_br. }}$. The other parameters remain the same as set 0 in 2.1. [a] The diffusion coefficient is normalized to $\mathrm{D}_{0, \mathrm{xx}}$ at $\mathrm{D}_{\text {rigid_br. }}[\mathrm{b}$ ] Diffusion coefficient index below reference rigidity $D_{\text {rigid_br. }}[c]$ Diffusion coefficient index above reference rigidity $D_{\text {rigid_br. }}$.

Table 2. Set 1 parameters for a break in the diffusion coefficient.

\begin{tabular}{cc}
\hline Parameter & Set 1 \\
\hline $\mathrm{D}_{0, \mathrm{xx}}\left[10^{28} \mathrm{~cm}^{2} \mathrm{~s}^{-1}\right]^{\mathrm{a}}$ & 25 \\
$\delta_{1}{ }^{\mathrm{b}}$ & 0.494 \\
$\mathrm{D}_{\text {rigid_br }}[\mathrm{GV}]$ & 200 \\
$\delta_{2}{ }^{\mathrm{c}}$ & 0.30 \\
\hline
\end{tabular}

Set 2: This run adds the second breaks starting from rigidities at $304 \mathrm{GV}$ for proton and Helium, $103 \mathrm{GV}$ for electron, $200 \mathrm{GV}$ for other elements, and have a change of primary nucleus injection indices at the breaks, with all the parameters listed in the table below. In the default parameter set, set $0^{7}$, the nucleus injection of Helium, proton, and electron each has individual nucleus injection indices. Therefore, this change only applies directly to the source spectra of all isotopes except for Helium, proton, and electron. As summarized in Table 3, second breaks are added to the nucleus at a rigidity of $R_{2}$. The Helium nucleus injection index $\gamma_{2, \mathrm{He}}$ is obtained by subtracting $\Delta_{\mathrm{He}}$ from the proton index $\gamma_{2, \mathrm{H}}$. The other parameters remain the same as set 0 in $\mathbf{2 . 1}$. [a] The nucleus injection index above the second rigidity break.

Table 3. Set 2 parameters for second breaks in the source spectra.

\begin{tabular}{cc}
\hline Parameter & Set 2 \\
\hline$\gamma_{2}{ }^{a}$ & 2.10 \\
$\mathrm{R}_{2}[\mathrm{GV}]$ & 200 \\
$\gamma_{2, \mathrm{H}^{\mathrm{a}}}$ & 2.178 \\
$\mathrm{R}_{2, \mathrm{H}}[\mathrm{GV}]$ & 304 \\
$\gamma_{2, \mathrm{e}} \mathrm{a}^{\mathrm{a}}$ & 2.378 \\
$\mathrm{R}_{2, \mathrm{e}}[\mathrm{GV}]$ & 103
\end{tabular}

The GALPROP plots of B/C ratio and the spectra of electron, proton, antiproton, Helium, Lithium, Beryllium, Boron, Carbon, and Oxygen are compared with the observation data from $\mathrm{CREAM}^{8}$ and $\mathrm{AMS}^{9,10,11,12}$ for verification. 


\section{Data and Discussion}

The GALPROP results are shown in the graphs below with data from AMS and CREAM.

Our results on the B/C ratio for three different parameter sets are compared in Fig.2. The set 0 and set 2 has a slight shift from the data, but it is still within the acceptable range. By adjusting the diffusion coefficient in set 1 , we can obtain a better fit of B/C ratio with GALPROP, thus set 1 shows a better curve of $\mathrm{B} / \mathrm{C}$ ratio.

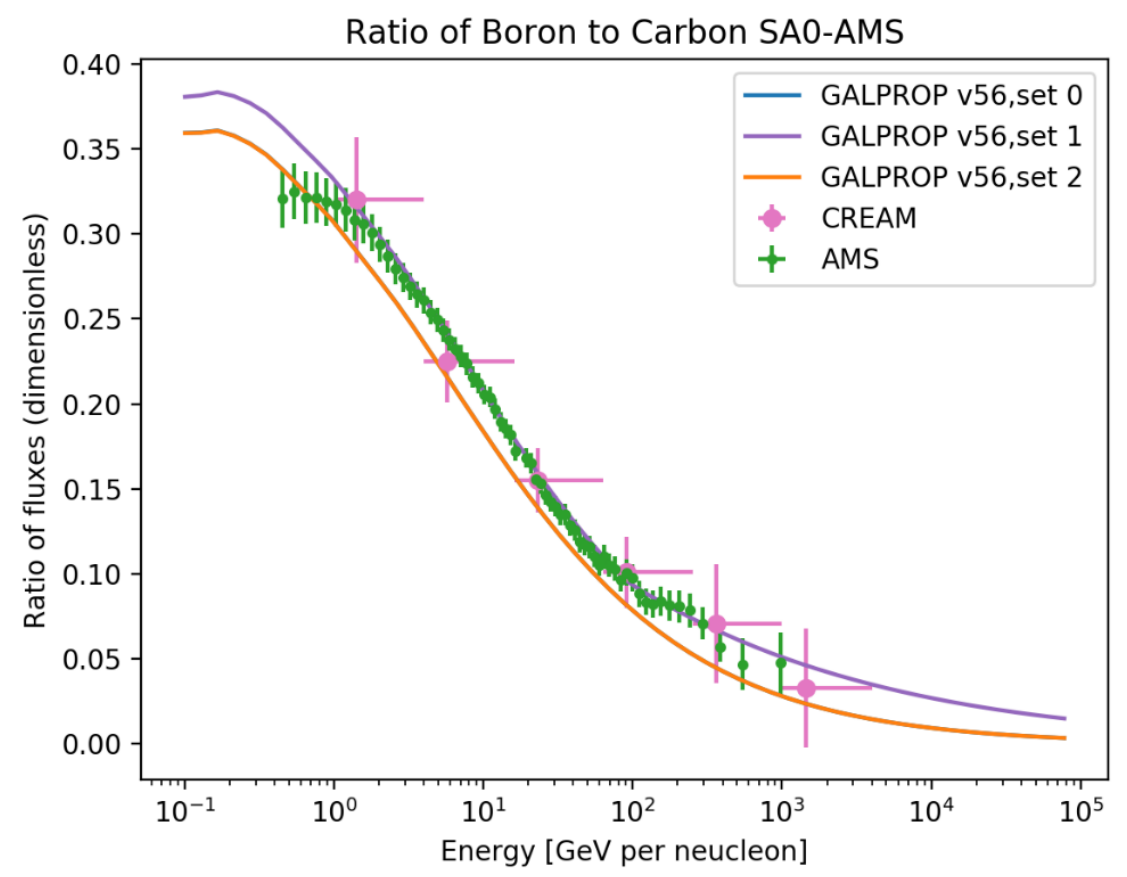

Figure 2: Continuous curves of B/C ratio produced by GALPROP v56 with data from CREAM ${ }^{8}$ and $A M S^{9}$. The set 0 curve (blue) is not visible because it coincides with the curve of set 2 (orange).

Figure 3 shows the spectra of $\mathrm{Be}, \mathrm{B}$, and $\mathrm{C}$. Both set 1 and set 2 can explain significant hardenings compared to set 0 to some extent, and agree better with the experimental data. The purple curve represents set 1 , which achieves a nice fit of all three elements. The orange curve represents set 2, which achieves a decent fit of Boron, but the hardenings in Carbon and Beryllium cannot be perfectly fitted, and is in general a less good fit.

Figure 4 shows the spectra of $\mathrm{He}, \mathrm{O}$ and Li. Both set 1 and set 2 explain the hardenings compared to set 0 for $\mathrm{O}$ and $\mathrm{Li}$ to some extent. The purple curve representing set 1 coincides with set 2 for Helium and shows a good agreement with the experimental data. Set 2 achieves a good fit of $\mathrm{O}$ and a less good fit of Lithium. Set 1 achieves better fits for both $\mathrm{O}$ and $\mathrm{Li}$ compared to set 2 .

Figure 5 shows the spectra of protons, electrons and antiprotons. The default fittings without breaks in set 0 cannot explain the significant hardenings in all three spectra. Set 1 has a good fit of proton and antiproton. The break in the diffusion coefficient cannot explain the hardening in the electron spectra, because the spectra of electrons mainly depend on the source spectra and the energy loss, instead of escape. Set 2 has a good fit of proton and electron, but is not enough to fit the hardenings in anti-proton spectra. 


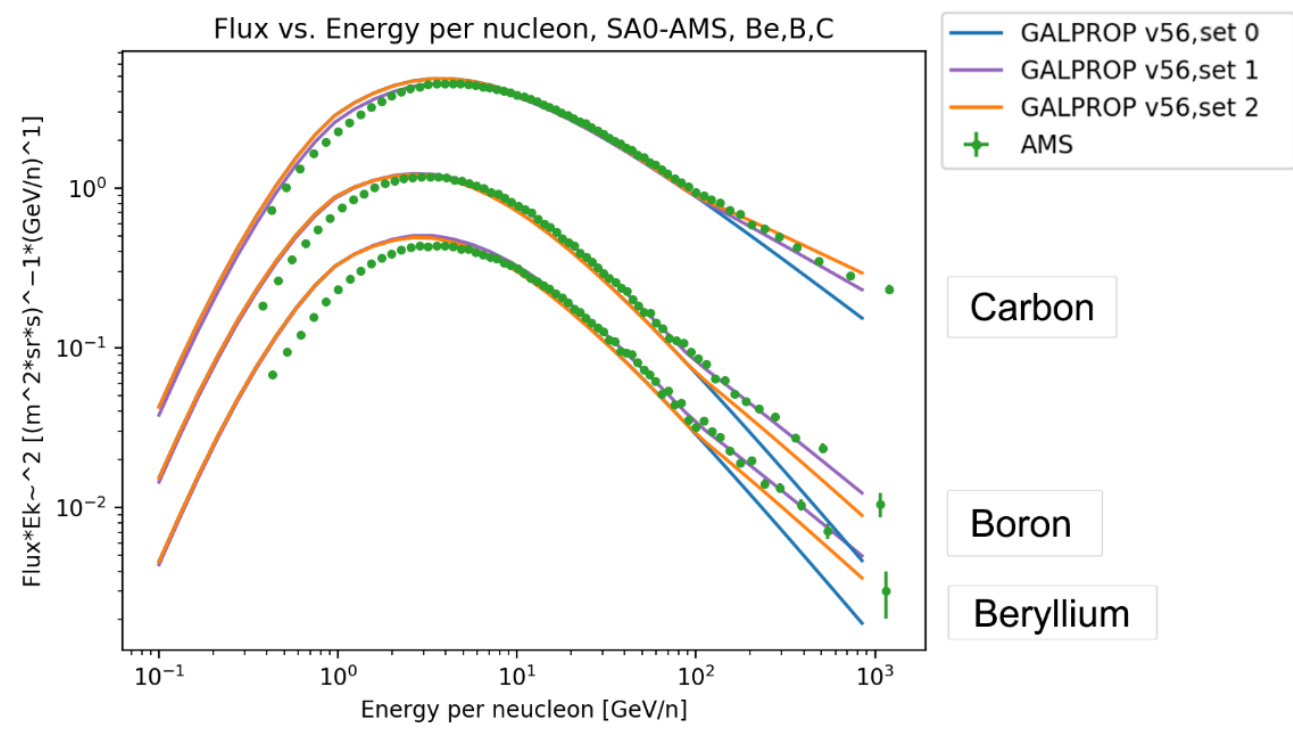

Figure 3: Continuous curves of Beryllium, Boron, and Carbon spectra produced by GALPROP v56 with data from $A M S^{9,13}$. The three collections of curves with data are arranged as the tags on the right indicate.
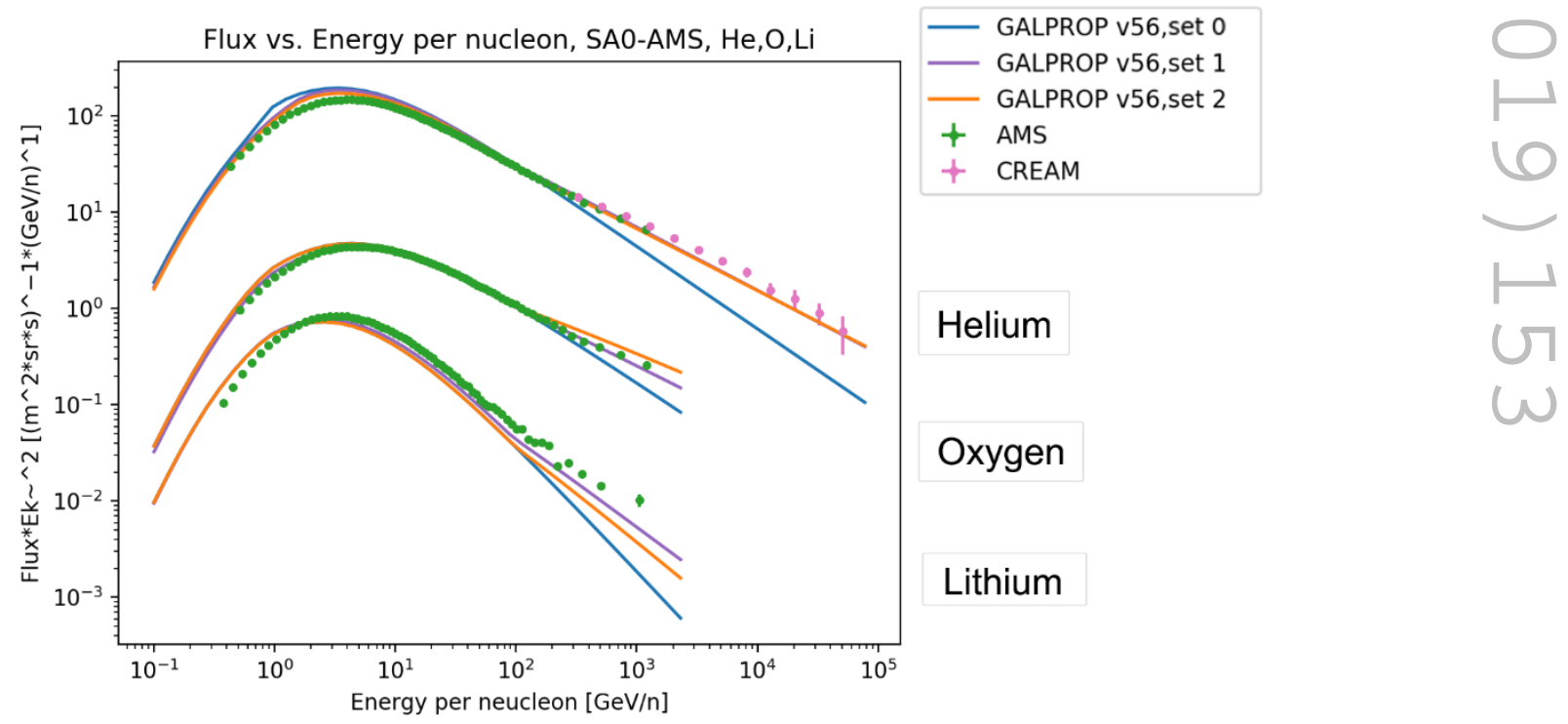

Figure 4: Continuous curves of Helium, Oxygen, and Lithium spectra produced by GALPROP v56 with data from CREAM ${ }^{8}$ and $A M S^{10,13}$. The three collections of curves with data are arranged as the tags on the right indicate. The set 1 curve of Helium (purple) is not visible at high energies because it coincides well with the curve of Helium of set 2 (orange). 


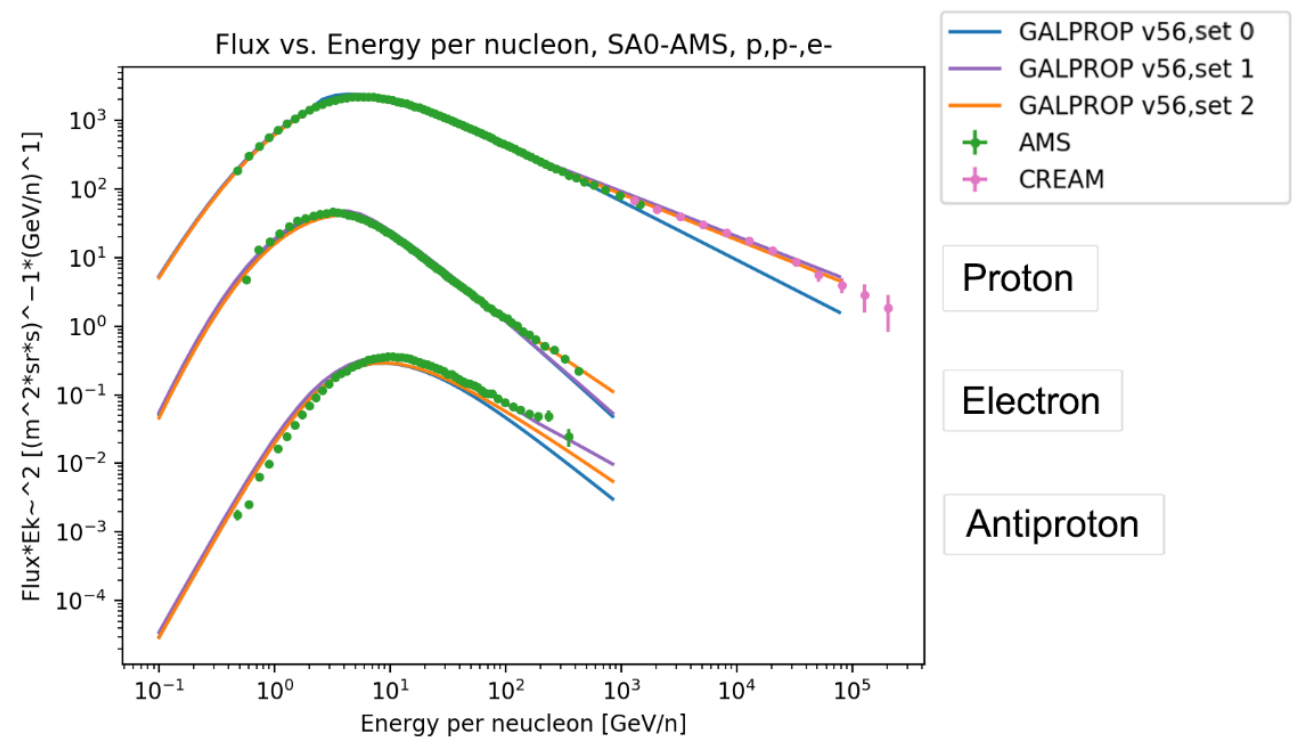

Figure 5: Continous curves of proton antiproton, and electron spectra produced by GALPROP v56 with data from $C R E A M^{8}$ and $A M S^{11,12}$. The three collections of curves with data are arranged as the tags on the right are shown. The set 2 curve of Proton (orange) coincides almost completely with the set 1 curve (purple). The set 0 curve of Electron (blue) is not very visible because it coincides well with the curve of set 1 (purple).

\section{Conclusion}

By using $D \propto \beta D_{0 x x} R^{-D_{g}},\left\{\begin{array}{l}D_{g}=0.494, \text { for } R \leq 200 G V \\ D_{g}=0.30 \text {, for } R>200 G V\end{array}\right.$, we add a break in the diffusion coefficient. While changing the diffusion coefficient index, GALPROP provides acceptable B/C ratio. The hardening in the spectra of $\mathrm{p}, \mathrm{p}-, \mathrm{He}, \mathrm{Be}, \mathrm{B}, \mathrm{C}$, and $\mathrm{O}$ are well fitted. The hardening in the Li spectra cannot be fitted perfectly, but it is improved from the default parameters without breaks in diffusion coefficient. The spectra of electrons are not well-explained, because the spectra of electrons rely mostly on the source spectra and energy loss in the galaxy instead of escape.

By applying $\Phi \propto R^{-\gamma},\left\{\begin{array}{c}\gamma=1.47, \text { for } R \leq 3.64 G V \\ \gamma=2.366, \text { for } 3.64 G V<R \leq 200 G V \text { to most elements, and } \\ \gamma=2.10, \text { for } R>200 G V\end{array}\right.$ applying specific source spectra breaks in electron, proton and Helium (parameters provided in Table 3), we add a break in the source spectra. Compared to changing diffusion coefficient at the rigidity break, changing the nucleus injection can also explain the hardenings in the proton and Helium spectra. While the change of the nucleus injection is enough to fit the electron curve, it is not enough for the hardenings in most primary and secondary elements.

These two methods still have weaknesses and cannot reach a perfect balance, but so far changing diffusion coefficient is a preferable way to explain the hardening in individual spectra and provide an acceptable Boron to Carbon ratio. However, the hardenings in electrons and some secondaries still need more minor changes on the parameters.

The possible changing of cosmic ray propagation parameters at rigidity $\sim 200 \mathrm{GV}$ was analyzed in the present paper in a simple semiempirical model. In principle, such an effect may 
occur for example in a model with different diffusion coefficients in the disk and halo of the galaxy ${ }^{14}$ and in a model where the outflow of cosmic rays from the galaxy is determined by their streaming instability at low energies and diffusion in the background interstellar turbulence at high energies ${ }^{15}$, see also ${ }^{5}$.

\section{References}

[1] Panov, A.D. et al. "Elemental energy spectra of cosmic rays from the data of the ATIC-2 experiment”, Bull.Rus.Acad. Sci. Phys, 71, 494 (2007).

[2] Ahn, H.S. et al. "Discrepant hardening observed in cosmic-ray elemental spectra", Astrohys. J. Lett, 714, L89 (2010).

[3] Adriani, O. et al. "PAMELA Measurements of Cosmic-Ray Proton and Helium Spectra", Science, 332, 69 (2011).

[4] Aguilar, M. et al. "Precision Measurement of the Proton Flux in Primary Cosmic Rays from Rigidity $1 \mathrm{GV}$ to $1.8 \mathrm{TV}$ with the Alpha Magnetic Spectrometer on the International Space Station". Phys. Rev. Lett., 114, 171103 (2015).

[5] Serpico, P., "Entering the cosmic ray precision era", J. Astrophys. Astr.onomy", 39, id41, 8pp (2018).

[6] https://galprop.stanford.edu/webrun/

[7] Porter, T. A., Jóhannesson, G. \& Moskalenko, I. V. High-energy Gamma Rays from the Milky Way: Three-dimensional Spatial Models for the Cosmic-Ray and Radiation Field Densities in the Interstellar Medium. ApJ 846, 67 (2017).

[8] Ahn, H. S. et al. Measurements of cosmic-ray secondary nuclei at high energies with the first flight of the CREAM balloon-borne experiment. Astroparticle Physics 30, 133-141 (2008).

[9] Aguilar, M. et al, Precision Measurement of the Boron to Carbon Flux Ratio in Cosmic Rays from $1.9 \mathrm{GV}$ to $2.6 \mathrm{TV}$ with the Alpha Magnetic Spectrometer on the International Space Station. Phys. Rev. Lett. 117, 231102 (2016).

[10] Aguilar, M. et al, Precision Measurement of the Helium Flux in Primary Cosmic Rays of Rigidities $1.9 \mathrm{GV}$ to $3 \mathrm{TV}$ with the Alpha Magnetic Spectrometer on the International Space Station. Phys. Rev. Lett. 115, 211101 (2015).

[11] Aguilar, M. et al, Precision Measurement of the Proton Flux in Primary Cosmic Rays from Rigidity $1 \mathrm{GV}$ to $1.8 \mathrm{TV}$ with the Alpha Magnetic Spectrometer on the International Space Station. Phys. Rev. Lett. 114, 171103 (2015).

[12] AMS Collaboration et al. Antiproton Flux, Antiproton-to-Proton Flux Ratio, and Properties of Elementary Particle Fluxes in Primary Cosmic Rays Measured with the Alpha Magnetic Spectrometer on the International Space Station. Phys. Rev. Lett. 117, 091103 (2016).

[13] Aguilar, M. et al. Observation of the Identical Rigidity Dependence of He, C, and O Cosmic Rays at High Rigidities by the Alpha Magnetic Spectrometer on the International Space Station. Phys. Rev. Lett. 119, 251101 (2017).

[14] Tomassetti N. Origin of the Cosmic-Ray Spectral Hardening. ApJ 752, L13 (2012).

[15] Blasi, P. et al. Spectral Breaks as a Signature of Cosmic Ray Induced Turbulence in the Galaxy. Phys. Rev. Lett. 109, 061101 (2012). 